\title{
Urgences
}

\section{Littérature et espace : une relecture du Quatuor d'Alexandrie}

\section{Jacques Pelletier}

Numéro 17-18, octobre 1987

L'esprit des lieux

URI : https://id.erudit.org/iderudit/025430ar

DOI : https://doi.org/10.7202/025430ar

Aller au sommaire du numéro

Éditeur(s)

Urgences

ISSN

0226-9554 (imprimé)

1927-3924 (numérique)

Découvrir la revue

Citer ce document

Pelletier, J. (1987). Littérature et espace : une relecture du Quatuor

d'Alexandrie. Urgences, (17-18), 157-168. https://doi.org/10.7202/025430ar d'utilisation que vous pouvez consulter en ligne.

https://apropos.erudit.org/fr/usagers/politique-dutilisation/ 


\title{
Jacques Pelletier \\ LITTÉRATURE ET ESPACE: UNE RELECTURE DU QUATUOR D'ALEXANDRIE
}

*Je voudrais faire un livre qui rève*. Arnauti dans Justine.

Mais la réussite eut été assurée s'il eút encore été possible de retenir cet instant précis, cet unique instant dans le temps et l'espace. s'il eût été possible de retenir l'existence palpable quil'environnait, la permanence pierreuse des murs, des dallages, de la maison, de la ville, toutes ces choses solidement assises et pourtant flottantes, cette envolée dans l'immobilité: de retenir cette oscillation sismique pénétrant tout cela et sur laquelle on glissait comme dans une barque sur un miroir d'eau, confondant son reflet avec celui de la lumière de midi, devenu livide en cet instant. Hermann Broch, La mort de Virgile.

\begin{abstract}
Le temps luj-mème, fluide fluide, n'est qu'une éponge souillée par toutes les entreprises qu'il semble encourager et qu'il enterre, les unes après les autres, dans un méme bain de tistesse. Le temps est une vierge enceinte. Et si c'est un fleuve, ce fleuve est un cimetière rapide qui emporte tout, mème les berges quil'étreignent, les arbres qui le bordent, les barques qu'il porte. Hubert Aquin. Neige noire.
\end{abstract}

Quand je lis, je suis ailleurs. Durant le temps de la lecture en effet, je prends congé de mes préoccupations quotidiennes, j'instaure une distance par rapport à la réalité qui constitue la trame de mes jours. Je décroche et, d'une certaine manière, je pars en voyage: je participe d'un autre lieu et d'un autre temps. Mon monde à moi, provisoirement, est aboli. Encore qu'il ne s'agisse pas tout à fait de cela, car ce monde créé par un autre c'est aussi le mien: c'est mon souffle de lecteur qui le fait vivre. C'est dire que tout à la fois, je suis dépaysé et renvoyé à moi-même. Car ce que je trouve dans un livre, c'est aussi - et surtout - ce que j'y mets. Et s'il me touche, c'est parce qu'en quelque part je peux me retrouver dans les lieux qu'il évoque et dans les émotions qui font vibrer les personnages qui l'habitent. Ainsi, c'est largement par des processus d'identification et de projection que je pénètre vraiment dans un texte ou, si l'on préfère, que celui-ci entre dans ma vie et contribue à sa façon à l'éclairer, sinon à la transformer. Ce n'est donc pas un mince pouvoir que possède la littérature mais elle ne l'exerce que si j'y consens.

C'est ce à quoi je songe au sortir de ma dernière lecture du Quatuor d'Alexandrie. Une lecture de commande effectuée pour les fins de la rencontre qui nous réunit ici, dans un lieu où j'ai passé neuf ans de ma vie, où j'ai connu des gens, entretenu des amitiés et des solidarités, où je reviens pour la troisième fois depuis mon départ, heureux d'y être mais conscient que je ne retrouverai pas le climat que j'ai connu à l'époque: le temps file, nous entraîne dans sa course 
folle, les choses changent ou du moins la perception que nous en avons - c'est là un thème durrellien sur lequel je reviendrai tantôt - et nous n'avons guère le choix, comme on dit, que de faire avec.

J'ai relu Le Quatuor donc, d'une traite, en trois jours, et le charme à nouveau a opéré: j'irais presque à dire que cette lecture m'a "réconcilié» avec la littérature, la grande, celle qui participe de la magie, qui vous donne du bonheur, qui met du soleil dans la grisaille des jours. Oui, je l'ai relu à peu près dans les mêmes conditions que lors de ma première lecture à dix-sept ans: dans l'éblouissement et le plaisir (du texte, comme dirait l'autre). Puis il y a eu une seconde lecture quelques années plus tard lorsque j'étais étudiant en lettres: une lecture plus professionnelle en quelque sorte, dans laquelle le roman était devenu un objet d'analyse, matière première d'un travail à remettre dans un cours, qui devait peu de temps après être repris sous forme d'article, premier texte de fond que j'ai publié dans la revue Études littéraires, en 1970 je crois. Cet article a trouvé un prolongement dans un petit livre écrit en 1974, publié en 1975, travail qui a nécessité une troisième lecture. Cette lecture effectuée au printemps 1974 a été la dernière - extensive j'entends - avant celle que je viens tout juste de refaire. Treize ans ont passé durant lesquels j'ai lu ce que Durrell a produit depuis Le Quatuor, et notamment son autre grande saga, le Quintette d'Avignon, sorte de re-make du Quatuor avec comme figure centrale à nouveau un lieu, une ville, Avignon, symbole de la Provence où, on le sait, Durrell a posé ses pénates au milieu des années 1950 , après avoir parcouru la planète durant des années comme chargé de mission à des titres divers pour le gouvernement britannique.

Comment expliquer l'engouement, l'envoûtement des lecteurs pour ce roman? Car c'est un phénomène que j'ai eu l'occasion de constater au fil des années: il existe quelque chose comme un fan club, non structuré, informel bien sûr, autour de Durrell en tant qu'auteur du Quatuor d'Alexandrie: un fan club réunissant des personnes qui, par de nombreux côtés, se ressemblent et souvent se rencontrent, entrent en communication par le truchement de leur passion pour le roman de Durrell: un fan club donc, ou si l'on préfère, une franc-maçonnerie fondée sur une complicité profonde, ce qui n'est pas un mince indice du rayonnement de ce livre.

À quoi cela tient-il? J'aurais tendance à croire que cette fascination relève, dans une large mesure, du statut même de ce roman, innovateur sur le plan de la forme, de sa conception d'ensemble, de l'utilisation extrêment habile que son auteur fait de la technique de la variation des points de vue, mais non révolutionnaire, ne rompant pas 
complètement avec le modèle du grand roman classique, notamment sous sa forme canonique du récit d'apprentissage. Et, par ailleurs, sur le plan du contenu, Le Quatuor intègre toute une imagerie romantique (mise en scène d'intrigues amoureuses plus ou moins mélodramatiques, d'affaires policières et politiques, évocation de moeurs et de traditions exotiques - celles de l'Égypte traditionnelle à travers notamment le personnage de Narouz) à une quête, une méditation sur l'amour moderne et sur le sens de l'existence, avec en prime une réflexion poético-philosophique sur les pouvoirs et les limites de la connaissance du coeur humain. Il y a donc matière à trouver son bien à la fois pour les spécialistes de la littérature (et particulièrement les narratologues), pour les amateurs d'aventure et d'exotisme, pour les passionnés de l'amour, enfin pour ceux que le problème de la connaissance intéresse. Sans compter la fascination qu'exerce l'évocation d'Alexandrie, dont le nom seul suffit à faire rêver.

Les lieux, on le sait, sont d'une importance extrême pour Durrell. Il croit en leur pouvoir, en leur rayonnement. Il ne s'agit pas pour lui de purs objets de contemplation, de rêverie, de simples cadres en somme. Il estime qu'ils sont à la source des comportements, tant individuels que collectifs. Dans L'esprit des lieux, l'ouvrage qui a inspiré le thème de notre rencontre, il écrit notamment:

Au fur et à mesure que vous apprenez à connaitre l'Europe, en appréciant les vins, les fromages et les gens des différents pays, vous commencez à vous apercevoir que le facteur déterminant de toute culture est, finalement, l'esprit des lieux. Tout comme un vignoble particulier vous donnera toujours un certain vin aux caractérisques reconnaissables, un pays comme l'Espagne, ou l'Italie, ou la Grèce, vous donnera le même type de culture et s'exprimera aussi bien à travers les individus qu'à travers ses fleurs sauvages'. Et plus loin: Oui, les êtres humains sont des expressions de leurs paysages, mais pour atteindre les sources secrètes d'une essence nationale, il vous faut quelques moments de solitude tranquille. En vérité, si elle était développée scientifiquement, la connaissance profonde des paysages pourrait nous fournir une science politique, car la moitié des décisions politiques prises dans le monde se fonde sur ce que nous appelons le caractère national ${ }^{2}$.

Et dans Justine, il fera écrire à Darley: «Maintenant, cependant, je commence à croire que le désir est hérité du site; que l'homme dépend pour ce qui est de cet accessoire qu'est la volonté, de sa situation dans un lieu; qu'il n'est qu'un locataire de terres fertiles ou de forêts malsaines» ${ }^{3}$.

Dans cette perspective on comprend toute l'importance qu'auront dans son oeuvre des lieux comme Alexandrie et Avignon, villes réelles mais aussi foyers de rêverie. Sur l'Alexandrie réelle que Durrell 
a connue durant la guerre comme agent d'information du gouvernement britannique, on trouve, tant dans sa correspondance avec Miller que dans L'esprit des lieux, des jugements extrêment durs. Ainsi il écrit en mars 1944, à une amie, Diana Gould:

\begin{abstract}
Entre-temps, partout la poussière et la saleté: les visages des Arabes avec leur veulerie et leur cupidité. Les tristes appétits fades des gens d'Alexandrie se répandent sur tout comme le son de poupées crevées. Les hululements stridents des femmes noires, le deuil qui se traduit par les cheveux arrachés, les vêtements lacérés - occupation qui nécessite de l'adresse - devant l'hôpital blanchi à la chaux. Le fez, le costume sombre, les bagues, l'accent français; la scrofule, la vérole, les richesses, la nourriture. Même dans votre bordel italien, je ne sais comment vous écrire ou vous parler depuis ce lieu de luxure, de perdition, ce creuset de l'ennui'.
\end{abstract}

Échappent à cette condamnation brutale les femmes, que l'écrivain trouve «[...] splendides comme des jardins négligés. Riches teintes de soie et d'olive, des yeux noirs fondus et des lèvres douces et arrondies, et des silhouettes célestes comme dans les dessins d'un Matisse sexuel ${ }^{5}$. Mais même cette fascination a des limites, Durrell considérant qu" "On ne peut rien trouver de plus agréable et de plus vide qu'une fille d'Alexandrie. Leur vide lui-même est comme une caresse $»^{6}$. De ces témoignages écrits sur le vif se dégage donc une perception globalement négative qu'on ne retrouvera pas telle quelle dans le roman qui sera écrit; il est vrai, dix ans plus tard. Avec suffisamment de distance donc pour qu'un travail se fasse sur le plan de la mémoire, de la perception, de l'imaginaire. De ce travail du temps le roman porte la trace et la marque.

Avant d'examiner comment la ville est représentée dans le roman, il importe de s'arrêter sur la conception qu'en a Durrell et qu'on retrouve formulée d'une part par Miller dans sa préface à Justine et, d'autre part par Darley à différents endroits du Quatuor. Pour Miller la ville est plus qu'un décor: c'est rien de moins qu'un personnage, une «entité vivante, un être quelque peu monstrueux fait de chair, de pierre, de crime, de rêve ou de mythe" ${ }^{7}$. Darley, quant à lui, narrateur principal de la plus grande partie du roman écrit de la ville qu'elle "se servit de nous, la ville dont nous étions la flore, la ville qui jeta en nous des conflits qui étaient les siens et que nous imaginions être les nôtres" ${ }^{8}$.

La description qu'en fournit Darley, par ailleurs, est faite de l'extérieur, à partir d'une île de la mer Égée où il a trouvé refuge après son départ d'Alexandrie. Description donc distanciée à la fois sur le plan spatial et sur le plan temporel: il s'agit en effet d'évoquer une ville en après-coup, sur le mode du souvenir et du rêve pour comprendre 
le sens de la partie de sa vie qui s'est jouée là et pour se débarrasser de la fascination qu'elle continue d'exercer sur soi. Entreprise d'exorcisme donc amorcée en vue de l'accession à la maturité: devenir enfin un homme et un artiste, objectif qui sera atteint au terme de la quête qui sert de fil conducteur, d'axe central du roman.

Ceci étant, comment la ville est-elle d'abord mise en scène? On en trouve une première évocation dès le début de Justine sur le mode d'une impression d'ensemble: «Dans un éclair, écrit Darley, je revois un millier de rues où tourbillonne la poussière. Des mouches et des mendiants en ont pris aujourd'hui possession, et tous ceux qui mènent une existence intermédiaire entre ces deux espèces ${ }^{9}$. La saleté donc, la poussière, la pauvreté. Et greffée là-dessus sa dimension mythique de "grand pressoir de l'amour" - pour reprendre une expression de Nessim -: «La provende sexuelle, précise Darley, qui est ici à portée de la main déconcerte par sa variété et sa profusion " ${ }^{10}$.

C'est ainsi que la ville réelle (dans le cadre de la fiction bien entendu) avec ses rues, ses quartiers, ses boutiques, ses cafés, ses restaurants, ses lieux de plaisir qui font l'objet de descriptions tout au long du roman, ne prend sa pleine signification que lorsque investie par le mythe dont la dimension centrale est la symbolique sexuelle. Darley, pour rendre compte de ce phénomène, distinguera ce qu'il appelle le "pôle réel» et le "pôle magnétique» de sa personnalité: "Son centre spirituel, écrit-il, était le site oublié de la Soma où le corps tourmenté du jeune soldat fut un jour placé dans sa divinité d'emprunt; son assise temporelle étant le Club des Courtiers où, tels des Gnostiques, les courtiers en coton venaient boire leur café, fumer des cigares rances et regarder Capodistria, comme des badauds des quais regardant un pêcheur ou un peintre» ${ }^{11}$.

Ce fameux "pôle magnétique» on en trouve une manifestation dans l'activité foisonnante des sectes, dans la prolifération des cultes religieux qui s'accommodent très bien par ailleurs du libertinage, d'un débordement sexuel effréné dans une sorte de "syncrétisme" qui apparaît aller de soi à Balthazar, personnage-clef de la ville, "médiateur entre les dieux et les hommes de la cité « ${ }^{12}$, comme le définit Darley à qui il explique qu' «il faut tenter de réconcilier les deux pôles d'habitudes et de comportements qui ne proviennent pas des dispositions intellectuelles des habitants, mais du sol, de l'air, du paysage. Sensualité débordante et ascétisme intellectuel ${ }{ }^{13}$. Plus loin, Darley se demandera pour sa part quelle ville au monde peut offrir un tel amalgame d'amour vénal et de mysticisme. Mais si la sexualité est partout présente, il ne faut pas se méprendre, c'est qu'elle n'a au fond guère d'importance, qu'elle n'a aucun caractère sacré: comme l'ex- 
prime crûment Darley: la ville «n'accorde guère plus d'importance aux femmes qu'à la nourriture [...] on les estime autant, disons, qu'un plat de mouton" et celles-ci à l'en croire, "réclament à grands cris qu'on abuse d'elles'" ${ }^{14}$.

Sexualité débordante donc, mais se déployant sur un fond de solitude et de tristesse et dont un cas de figure tragique est mis en relief dans le personnage de Melissa, amante de Darley, maîtresse du vieux et riche Cohen, modèle puis danseuse dans un cabaret de bas étage, qui répondra à l'écrivain Pursewarden qui lui demande comment elle se défend contre la solitude: «Monsieur, je suis devenue la solitude même ${ }^{15}$. Autrement dit, j'existe mais je ne vis pas, sinon sur le mode de l'absence: je suis un désert, j'ai fait le deuil de moi-même, je suis morte: ce que vous voyez, c'est l'ombre d'une ombre, un résidu, une ruine dont il ne restera bientôt plus de traces. C'est cette réplique qui séduira Pursewarden, lui-même personnage tragique dont la vie n'est qu'un combat pour acclimater, aménager le désespoir qui le ronge inexorablement, comme un cancer. Mais c'est là une autre histoire et je reviens à la représentation de la ville que nous offre le roman.

Ce qui la caractérise également, c'est son cosmopolitisme. C'est la «seule ville au monde, écrit Darley, où les races et les coutumes les plus étrangères se mêlent et se marient, où les destins les plus intimes se recoupent ${ }^{16}$. Et le narrateur impersonnel et omniscient de Mountolive, troisième tome de la saga que Durrell qualifie de roman "strictement naturaliste» rapportant donc en principe la vérité des faits et des événements, ce narrateur précise:

\begin{abstract}
Alexandrie était encore l'Europe, la capitale de l'Europe asiatique, si un tel état est concevable. Elle ne serait jamais semblable au Caire, qui descend d'une lignée purement égyptienne et dont l'arabe est la langue essentielle; ici c'étaient le français, l'italien et le grec qui étaient le plus largement répandus. L'atmosphère, les coutumes sociales, tout était différent, coulé dans un moule européen où les chameaux, les palmiers et les indigènes en robe ne formaient qu'une frise haute en couleurs, la toile de fond d'une vie aux origines diverses ${ }^{17}$.
\end{abstract}

La véritable Égypte, d'une certaine manière, doit donc être cherchée ailleurs: au Caire, oui, mais aussi dans l'arrière-pays, notamment dans la propriété terrienne des Hosnani dont Darley comprend dans Balthazar, que "c'était vraiment là l'Égypte - une Égypte copte - tandis que la cité blanche, comme vue à travers un prisme sale, grouillait d'images troublantes et indésirables, venues de terres étrangères - des infiltrations de la Grèce, de la Syrie, de la Tunisie ${ }^{18}$. Ville bigarrée, hybride, bâtarde, ni vraiment arabe, ni vraiment européenne dont le caractère cosmopolite sert à fois d'aimant 
et de repoussoir, qui tour à tour attire, fascine et déçoit et que les principaux héros du roman quitteront à la fin de la saga, ayant pris une juste mesure de la ville, délivrés du lien passionnel qui les liait à elle.

Ce caractère mythique du lieu, Darley en aura une pleine conscience lorsqu'il reviendra à Alexandrie après la guerre et il l'exprimera dans un beau passage qui rend compte à la fois de cette découverte et du thème central du livre qui est, comme on le sait, la quête de l'amour moderne:

Nous étions trois écrivains, livrés à une ville mythique dont nous devions tirer substance, au sein de laquelle nous devions confirmer nos dons. Arnauti, Pursewarden, Darley - tels le passé, le présent et le futur d'un même verbe! Et de même dans ma propre vie (ce courant qui s'écoulait mollement du flanc blessé du Temps!), trois femmes se disputaient les humeurs du grand verbe Amour:

Melissa, Justine et Cléa'19.

De retour à Alexandrie, c'est par conséquent un Darley transformé qui renoue avec une ville qu'il avait re-construite, comme il le dit au début de Cléa, selon son esprit et son coeur et qu'il ne retrouve pas sur place telle qu'il l'avait rêvée: "Nous nous abusons toujours, écrit-il, en croyant retrouver les êtres et les choses inchangés. L'Alexandrie qui se présenta à mes yeux, la première vision que j'en eus de la mer, jamais je n'aurais pu imaginer cela ${ }^{20}$ Et plus loin: «Pour ma part, je la voyais maintenant telle qu'elle avait toujours dû être: un petit port modeste établi sur un banc de sable, une mare stagnante, moribonde et sans âme " ${ }^{21}$. Cette perception renouvelée est liée à son amour pour Cléa: "Si l'on est amoureux d'un de ses habitants, une ville contient tout l'univers. Toute une nouvelle géographie d'Alexandrie se découvrait à travers Cléa, ravivant d'anciennes significations, rajeunissant des sensations à demi oubliées, déposant comme une nouvelle couche de couleur vive sur une ancienne histoire, une ancienne biographie ${ }^{22}$.

Ces passages indiquent très clairement, il me semble, que le roman de Durrell, contrairement aux prétentions de son auteur, n'est d'abord pas sans doute un livre sur un lieu, un espace - bien qu'il soit aussi cela - mais un livre sur le temps, différent bien évidemment du roman de Proust dans la mesure où il ne se présente pas comme une recherche du temps perdu qu'on essaie de traquer et de figer dans l'intemporalité de l'art mais comme une exploration du passé guidée par des préoccupations d'avenir: faire revivre le passé, oui, mais pour s'en libérer et pouvoir repartir, vers d'autres lieux, d'autres amours, d'autres créations. En cela Le Quatuor est bien le roman de l'Espace- 
Temps, notion évoquée par Durrell dans sa note liminaire de Balthazar. Et cette préoccupation n'apparaît pas uniquement dans Cléa dont elle constitue le thème principal - mais déjà dans Justine lorsque Darley, de retour d'un séjour de deux ans en Haute-Égypte, découvre un Nessim changé: "ll avait vieilli comme une femme, écrit-il: ses hanches et son visages s'étaient épaissis " ${ }^{23}$ et une Justine que Cléa lui décrit dans une lettre de la manière suivante: «Et plus je la regardais, plus je me rappelais le merveilleux démon qu'elle avait été pour nous tous, et moins je comprenais comment elle avait pu se transformer en cette petite paysanne boulotte aux mains sèches et calleuses $»^{24}$. Constatation qui suscite chez elle la réflexion que «le temps nous emporte (...) nous pousse en avant ${ }^{25}$.

Ce thème est repris de manière exhaustive dans Cléa. Darley, de retour à Alexandrie, à la fin de la guerre, réalise cruellement qu'il ne se rappelle littéralement plus de Melissa, qu'il n'en conserve plus de souvenirs, si ce n'est celui de son nom:

\begin{abstract}
Avec surprise, avec tristesse aussi, je m’aperçus qu'elle avait entièrement disparu (...) C'était comme si elle n'avait jamais existé, comme si elle ne m'avait jamais inspiré la peine et la pitié qui (je me l'étais toujours dit) continueraient à vivre, transmuées en d'autres formes peut-être mais à vivre glorieusement, éternellement. Je l'avais usée, son image, comme une vieille paire de chaussette, et l'absolu de cette disparition me surprit et me révolta ${ }^{26}$.
\end{abstract}

Choc brutal en effet que de constater que le passé est irrécupérable, qu'il n'existe plus, même sur le mode du souvenir et que seule la fille de Melissa et de Nessim pourra témoigner qu'un jour, sans doute, Darley a aimé cette femme, Melissa, ce pauvre «oiseau blessé» comme on la décrit quelque part, cette "Notre-Dame des douleurs" au destin tragique.

Mais si Melissa est morte, les autres amis de Darley, bien vivants, apparaissent, eux, profondément transformés. Nessim, par exemple, Darley a du mal à le reconnaître derrière le bandeau noir qui recouvre l'oeil qu'il a perdu lors d'un bombardement et il lui paraît «beaucoup plus grand et plus maigre que l'image que j'avais gardée de lui ${ }^{27}$. Il en va de même pour Justine qu'il trouve amaigrie, encore belle mais de «la beauté passive d'une momie propertienne qu'on aurait maladroitement peinte pour donner l'illusion de la vie, ou d'une photographie colorée sans soin ${ }^{28}$. Et il comprend que Justine a été dans une très large mesure un produit de son imagination, de son amour:

Je voyais maintenant que ma Justine n'avait été rien de plus que l'oeuvre d'un illusionniste, fondée sur l'armature défectueuse de paroles, d'actes et de gestes mal interprétés. Il n'y avait là rien de répréhensible en vérité; 
le véritable coupable, c'était mon amour qui avait inventé une image dont il se nourrissait. Il n'était pas davantage question de malhonnêteté, car l'image était colorée selon les nécessités de l'amour qui l'avait inventée $\mathrm{e}^{29}$.

Darley découvre donc tout à la fois la vérité sur Alexandrie, ce modeste port de mer qu'il a magnifié en en faisant une capitale de rêve, un lieu magique, la capitale de la mémoire dans Justine et Balthazar et sur sa maîtresse privilégiée d'alors dont il était amoureux de l'image qu'il s'en était lui-même fabriquée. La femme qu'il a aimée, ce n'était sans doute par la Justine réelle, mais son amour ne pouvait exister sans la représentation mythique de celle-ci. Sans magie, pas d'amour, ou à tout le moins pas de passion: au mieux de l'affection et de la tendresse.

C'est l'un des effets du temps que de faire s'évaporer la magie: c'est en quoi il est destructeur. C'est la prise de conscience que Marcel fait au terme de La Recherche, notamment à propos de Combray, ce lieu qui, durant l'enfance, semblait immense et qui, au fil des années, s'est ratatiné comme une peau de chagrin jusqu'à n'apparaître plus à l'âge adulte que comme un modeste et insignifiant petit village de la France profonde; c'est cela que l'on retrouve aussi dans le "bon côté des choses", le royaume féérique de l'enfance de Tinamer, dans l'Amélanchier de Ferron. Cette expérience, nous la refaisons tous, pour notre propre compte, lorsque nous retournons sur les lieux de notre enfance: cet endroit qui dans nos souvenirs est grandiose, nous constatons non sans effroi que ce n'est rien: une petite maison, une courette, et nous réalisons que le temps a passé et qu'on ne le rattrapera plus, qu'il n'y a pas d'autre choix possible que d'assumer le présent, que la vie est un train dont le billet est à aller simple seulement et qu'il fonctionne à un très haut régime, nous entraînant dans sa course frénétique vers la mort, le néant et éventuellement, pour ceux qui y croient, vers notre renaissance.

La renaissance, c'est ce que, d'une certaine manière, Darley trouve avec Cléa, succédant dans sa vie amoureuse à Melissa et à Justine. Cette Cléa Montis, un peu effacée de Justine, «eau morte du chagrin" comme la décrit une note de l'appendice du premier tome du Quatuor - Effet d'intertextualité ou projection de ma part? Aquin, dans Neige noire, évoque les «muscles du chagrin» de Sylvie Dubuque-Lewandowsky - qu'il redécouvre «beaucoup plus belle que le souvenir que j'avais gardé d'elle, plus mince, avec une gamme subtile de nouveaux gestes et de nouvelles expressions qui suggéraient une nouvelle et troublante maturité ${ }^{30}$. Cléa avec qui il vivra un autre amour, fondé sans doute sur le désir, mais aussi sur autre chose: l'amitié, la complicité, nouvelle forme d'amour succédant à l'amour- 
charité vécu avec Melissa et à l'amour-passion éprouvé pour Justine. Cette maturité de l'homme et de l'artiste se traduira par son départ d'Alexandrie:

\begin{abstract}
Je sens, écrit-il, que cette ville s'estompe en moi, qu'elle se déprend de mes pensées, comme un mirage qui s'éloigne, comme la triste histoire d'une grande reine dont le destin s'est perdu dans la déroute des années et les sables du temps! Mon esprit se tourne de plus en plus vers l'Occident, vers le vieil héritage de l'Italie ou de la France. Il y a peut-être encore bien des trésors à retirer de leurs ruines ${ }^{31}$.
\end{abstract}

Cléa, de même, quittera la ville blanche pour la France, convaincue que ce n'est que là qu'elle pourra se réaliser en tant qu'artiste-peintre, congédiant sa ville natale qui «maintenant, écritelle, a perdu tout attrait à mes yeux" ${ }^{32}$ mais non sans éprouver une certaine nostalgie: «Mais pouvons-nous, ajoute-elle, nous empêcher de chérir en secret les lieux qui nous ont fait le plus souffrir? ${ }^{33}$ Enfin, Mountolive, l'ambassadeur, quittera également le Caire et Alexandrie pour Paris et Justine et Nessim, engagés dans un nouveau projet, transfigurés, paraissant rajeunis, partiront, pour leur part, pour la Suisse.

Le roman se termine donc par une ouverture sur l'avenir. Au terme de son apprentissage amoureux et artistique réalisé au moyen du travail de re-création qu'il a opéré sur Alexandrie, Darley est un écrivain parvenu à maturité pouvant désormais écrire des fables commençant par le célèbre «ll était une fois...» de tous les conteurs de la planète. Sa carrière d'écrivain commence donc vraiment à la fin du roman qui, lui, témoigne du génie de Durrell.

Alors le Quatuor, un roman sur Alexandrie? Oui sans doute pour une part. Mais quelle Alexandrie? La ville égyptienne réelle, sise en un lieu précis de la géographie de ce pays? La ville imaginaire rêvée par Darley, narrateur principal du roman, travaillant à partir des observations et des souvenirs de Durrell? La ville comme personnage? Si l'on veut, mais comme personnage principal dont les êtres humains seraient des jouets? Alors là, non. Non pas que l'évocation du livre n'ouvre pas la porte à la rêverie, à l'imagination: je songe en particulier à certaines séquences dans le désert, au dépeçage des chameaux - cette scène atroce -, à la chasse du Magzub par Narouz, à la mort de celui-ci, à son enterrement scandé par les cris des pleureuses, à la visite de Mountolive au bordel d'enfants, au bal des Cervoni, à la pêche sur le lac Maréotis etc. Il ne s'agit pas de nier cela mais ce qui m'a le plus frappé à la relecture, ce sont les rapports entre les personnages, les rapports affectifs surtout, le discours amoureux qui circule tout au long du roman - dans lequel Barthes aurait pu 
trouver matière à nourrir quelques-uns de ses Fragments - , ce qui est dit sur la connaissance et sur l'art aussi, et enfin sur le temps.

Les lieux, au fond, sont affaire de décor: ils n'ont pas d'autre âme que celle que nous leur prêtons. Et personnellement ils ne m'intéressent pas vraiment. Pas en soi en tout cas. Seulement lorsqu'ils sont habités par des gens que j'aime ou s'ils symbolisent un style de vie qui me plaît. Ainsi si j'ai tant aimé Dubrovnick sur la côte adriatique, c'est que j'y ai séjourné avec une femme que j'aimais et des amis chers, que la mer au surplus était douce, qu'il faisait bon s'y plonger puis se faire sécher au soleil en buvant du bon vin. Et si la Provence me tient tant au coeur, c'est pour son ciel bleu d'acier, bien sûr, d'une luminosité qu'on ne retrouve vraiment que là et ici, parfois, un peu dans le Bas du fleuve et la Gaspésie, mais jamais en tout état de cause à Montréal où il est toujours recouvert, comme enveloppé par un voile, une mince pellicule blanchâtre, et c'est aussi pour la faconde de ses habitants et la nonchalance de leur style de vie. Et quand je vais à Paris ou ailleurs en Europe, à toute visite de haut lieu historique et culturel, j'avoue préférer la flânerie libre dans les rues, me fiant au hasard pour faire des découvertes, ou m'abandonnant tout simplement à humer l'air du temps. Aussi n'irai-je sans doute jamais à Alexandrie car je m'y rendrais pour faire la connaissance de Melissa, de Justine, de Cléa, de l'aimable binoclard Darley ou du désespéré écrivain Pursewarden, ces créatures de rêve qui n'existent que dans ma tête. Pour la ville, je me contenterai probablement toujours des images que fait naître en moi Durrell, craignant que la réalité ne soit bien en-dessous de la fiction. En me faisant croire cela, Durrell se révèle l'immense écrivain qu'il est, un des plus importants de l'époque, en dépit de sa théorie déterministe, mécaniste, et disons le, guère convaincante, des lieux.

\section{NOTES}

1. Lawrence Durrell: L'esprit des lieux, Paris, Gallimard, 1976, p.182.

2. Idem: p.184.

3. Lawrence Durrell: Justine, Paris, Buchet-Chastel, 1959, repris dans «Le livre de poche*, p.191.

4. L'Esprit des lieux, op. cit., p.83. Je souligne.

5. Une correspondance privée, Paris, Buchet-Chastel, 1963, p.201

6. Ibidem.

7. Justine, op. cit, p.7.

8. Idem: p.16.

9. Idem: p.17.

10. lbidem.

11. Idem: p.63.

12. Idem: p. 153

13. Idem: p.165-166.

14. Lawrence Durrell: Balthazar, Paris, Buchet-Chastel, 1959, repris dans «Le livre de poche*, p.228.

15. Lawrence Durrell: Mountolive, Paris, Buchet-Chastel, 1959, repris dans *Le livre de poche», p.265

16. Balthazar, op.cit., p.3.

17. Mountolive, op.cit., p 231-232.

18. Balthazar, op. cit. p.119.

19. Lawrence Durrell: Cléa, Paris, Buchet-Chastel, 1960, repris dans Le Ivre de poche*, p.275.

20. Idem: $\mathrm{p} 32$.

21. Idem: p.157-158 
22. Idem: p.356.

23. Justine, op. cit., p.406

24. Idem: p.411.

25. Ibidem.

26. Cléa, op.ctt., p.60

27. Idem: p.42.

28. Idem: p.74.

29. Idem: p.85.

30. Idem: p.119.

31. Idem: p.431-432.

32. Idem: $p .436$.

33. Ibidem. 